\title{
Vascular Imaging in Stroke: Comparative Analysis
}

\author{
Kristian Barlinn • Andrei V. Alexandrov
}

Published online: 21 June 2011

(C) The American Society for Experimental NeuroTherapeutics, Inc. 2011

\begin{abstract}
Advances in stroke treatment have mirrored advances in vascular imaging. Understanding and advances in reperfusion therapies were made possible by improvements in computed tomographic angiography, magnetic resonance angiography, neurovascular ultrasound, and renewed interest in catheter angiography. As technology allows better noninvasive vascular diagnosis, digital subtraction angiography (the remaining gold standard for vascular imaging) is increasingly used for rescue procedures and elective interventions. This review will examine specific advantages and disadvantages of different vascular imaging modalities as related to stroke diagnosis.
\end{abstract}

Keywords Vascular imaging · Stroke · MRA · CTA · TCD · Carotid duplex · DSA

\section{Introduction}

Our ability to diagnose and differentiate a stroke depends almost entirely on imaging and further determination of a specific pathogenic mechanism, which often relies on imaging of the pre-cerebral and cerebral vasculature. This chapter will

Electronic supplementary material The online version of this article (doi:10.1007/s13311-011-0042-4) contains supplementary material, which is available to authorized users.

K. Barlinn · A. V. Alexandrov $(\bowtie)$

Comprehensive Stroke Center,

University of Alabama at Birmingham Hospital,

Birmingham, AL 35249, USA

e-mail: avalexandrov@att.net

K. Barlinn

Dresden University Stroke Center,

University of Technology Dresden,

01307 Dresden, Germany review noninvasive and invasive tools available to clinicians for diagnostic work-up and monitoring of stroke patients.

\section{Noninvasive Vascular Imaging}

Magnetic Resonance Angiography

Time-of-flight (TOF) magnetic resonance angiography (MRA) and contrast-enhanced MRA (CE-MRA) are the most frequently used MRA techniques for the evaluation of cervical and intracranial arteries in patients with cerebrovascular disease. These techniques are based on gradient echocardiographic sequences with either 2-dimensional or 3-dimensional volume acquisition [1]. TOF-MRA does not require an injection of a contrast medium and therefore constitutes an alternative for patients who are unable to receive contrast. Even though spatial resolution with TOFMRA is superior to that in CE-MRA (especially 3dimensional TOF sequences), the volume covered is limited by vascular saturation artifact, thus making this technique more suitable for the evaluation of the intracranial vasculature (Table 1) [1, 2]. Since vascular signal is dependent on flow direction and velocity through the imaging plane, TOF-MRA tends to overestimate the degree, as well as length of stenosis (mainly in the presence of turbulence, slow or re-circulating/reversed blood flow). Therefore, the distal vasculature may not be accurately displayed [2]. Nevertheless, TOF-MRA has an acceptable sensitivity for the depiction of intracranial steno-occlusive lesions compared with digital subtraction angiography (DSA) and computed tomographic angiography (CTA). The less frequently used phase-contrast MRA may provide additional physiologic information like blood flow velocity and flow direction $[2,3]$. 
With CE-MRA, the passage of a gadolinium contrast bolus through the arteries of interest provides an angiographic effect, which is independent of blood flow, thus having fewer artifacts derived from turbulences and slow blood flow [1]. Image acquisition in CE-MRA is faster and provided coverage is larger. For these reasons, assessment of cervical carotid and vertebral artery disease (particularly high-grade stenoses and blood flow disturbances) is more accurate with CE-MRA than with TOF-MRA techniques $[4,5]$, and atherosclerotic carotid artery lesions amenable for carotid endarterectomy (CEA) can be more reliably differentiated (Fig. 1) [4].

Furthermore, CE-MRA may demonstrate a complete occlusion or stenosis of the arterial lumen in patients with cranio-cervical arterial dissections and improve the overall accuracy of magnetic resonance imaging (MRI) in the detection of this disease [6] if combined with T2 fatsuppression sequences.

Another application for MRA is the diagnosis, screening, and follow-up imaging of cerebral aneurysms, which can be detected by TOF-MRA techniques, depending on the overall size of the aneurysms (sensitivity increases with larger size aneurysms) and local hemodynamics $[5,7]$.

As a certain limitation, it should be mentioned that availability and feasibility of MRI is limited in acute stroke patients (Table 2) [8]. Some stroke patients cannot tolerate MRI or cooperate, poorly leading to movement artifacts. MRI/MRA cannot be used in patients with pacemakers, certain magnetic appliances, and those who have allergies to contrast media. In addition, there was a significant decline in the use of gadolinium, due to recent recognition of its rare complications, such as nephrogenic systemic fibrosis in patients with an impaired kidney function [9].

CTA

CTA is the most common first-line diagnostic modality for vascular imaging in the setting of acute stroke [10]. CTA is widely available, well tolerated by the majority of stroke patients, and with a single bolus of iodinated contrast, thinslice, high-resolution, volumetric spiral computed tomographic (CT) images of the pre-cerebral and cerebral vasculature, CTA can be obtained quickly after nonenhanced CT [11, 12]. In addition to axial CTA source images (CTA-SI), post-processing techniques for 3dimensional CTA imaging (e.g., maximum intensity projection, shaded surface display, and volume-rendering techniques) can be performed in few minutes and provide images comparable with those obtained with DSA (Fig. 1) $[13,14]$.

In acute ischemic stroke, CTA can reliably detect intracranial proximal arterial occlusions and stenoses (Table 1) [15]. The presence of a proximal arterial 


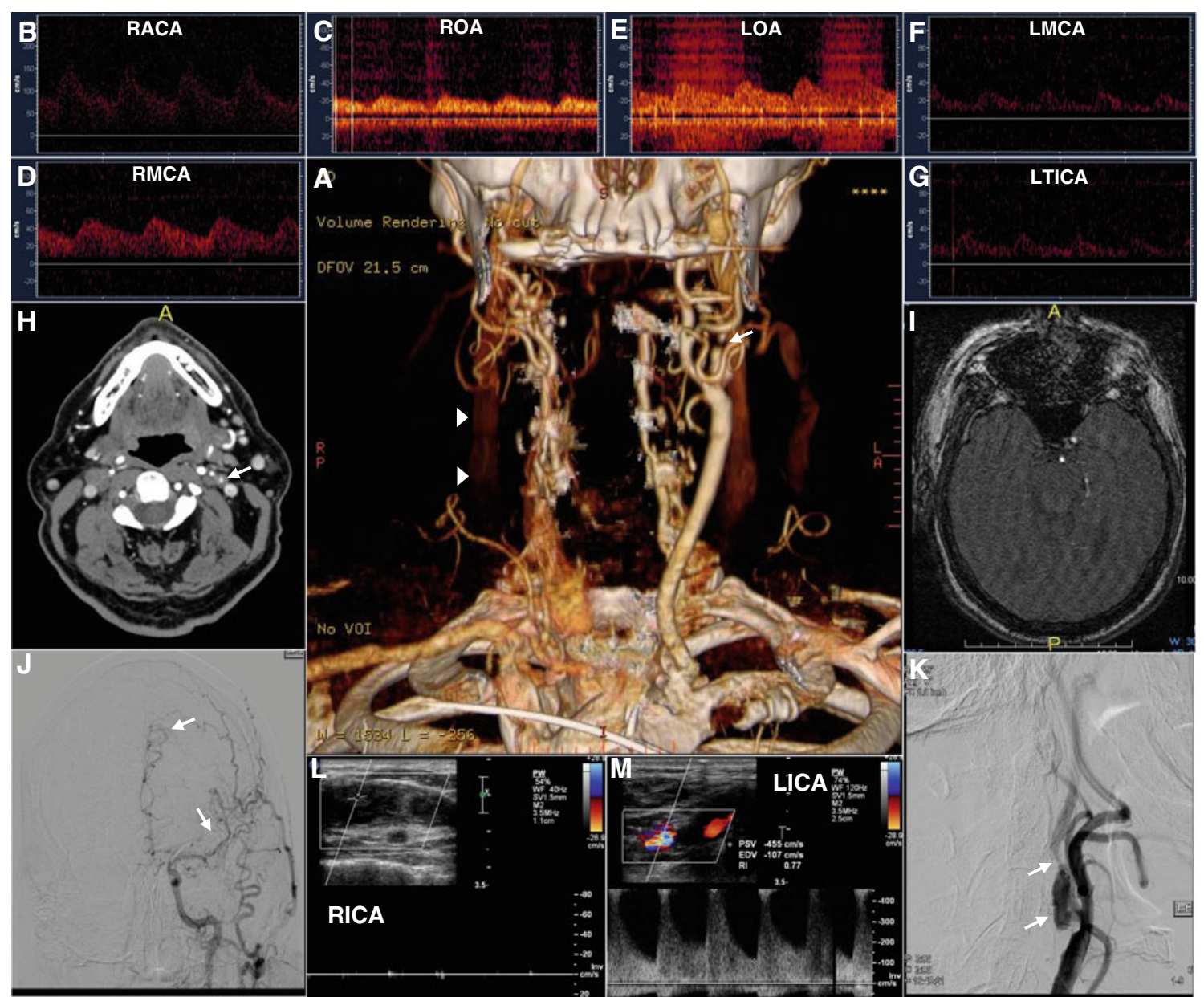

Fig. 1 a Computed tomographic angiography (CTA) (3-dimensional volume-rendering) shows a complete occlusion of the right common carotid artery (arrowheads), as well as near complete occlusion of the proximal left internal carotid artery (ICA) just after the bifurcation (arrow). b-d Transcranial Doppler (TCD) shows a blunted signal in the right middle cerebral artery (RMCA), reversed right ophthalmic artery (ROA) and anterior cross-filling from the left to right anterior cerebral artery (RACA) consistent with a hemodynamically significant obstruction in the right ICA. e-g Blunted waveforms in the left middle cerebral artery (LMCA) and terminal ICA (LTICA), as well as reversed left ophthalmic artery (LOA) suggestive of high-grade stenosis in the cervical ICA. h CTA source images demonstrates near occlusion of the left proximal ICA (arrow) and a complete occlusion of the right ICA. i Time-of-flight magnetic resonance angiography

occlusion on CTA predicts functional outcome, final infarct size, and response to intravenous thrombolysis, thus facilitating decision making for intra-arterial rescue procedures $[16,17]$. In addition, CTA-SI can provide information regarding the quality of collateral circulation and may improve the sensitivity to identify ischemic areas not seen on nonenhanced CT [18].

CTA is also highly accurate in determining the severity of atherosclerotic stenoses in the extracranial arteries, especially the carotid arteries, thus aiding selection of patients amenable for revascularization [19]. Furthermore, shows substantial motion artifacts, as well as an occlusive appearance of the right ICA with an abnormal flow signal in the remainder of the visualized left ICA. j, k Digital subtraction angiography reveals an aneurysmal dilatation of the proximal left ICA and a focal stenosis just distal to the aneurysm that measures approximately $75 \%$ by the North American Symptomatic Carotid Endarterectomy Trial (NASCET) criteria (K, arrows). Intracranial anteroposterior view $(J)$ with injection of contrast into the left ICA shows relatively poor flow into the left internal carotid artery system (arrows) attributable to the high-grade ICA stenosis. I, m Carotid duplex B-mode and color Doppler show evidence of a right internal carotid artery (RICA) occlusion with no detectable flow and a $>70 \%$ stenosis in the left internal carotid artery (LICA)

it allows a differentiation between near-occlusions and complete occlusions and is valuable in the diagnosis of an extracranial arterial dissection [20].

In comparison to DSA, CTA shows a high sensitivity and specificity in the assessment of intracranial aneurysms in patients with subarachnoid hemorrhage (SAH) [21], and CTA is deployed as the initial test of choice in the diagnostic work-up of patients with suspected aneurysms and subarachnoid hemorrhage. In addition, CTA is now increasingly being used in the diagnostic work-up of an underlying cause of a spontaneous intracerebral hemor- 
Table 2 Advantages and disadvantages of various vascular imaging modalities

\begin{tabular}{|c|c|c|c|c|c|}
\hline & MRA & CTA & Duplex & TCD & DSA \\
\hline \multirow[t]{5}{*}{ Advantages } & $\begin{array}{l}\text { - No contrast needed } \\
\text { (TOF) }\end{array}$ & - Widely available & - Inexpensive & - Inexpensive & • High-spatial resolution \\
\hline & $\begin{array}{l}\text { - Hemodynamic } \\
\text { information from the } \\
\text { aortic arch up to the } \\
\text { cerebral vessels }\end{array}$ & $\begin{array}{l}\text { - Rapid data } \\
\text { acquisition }\end{array}$ & - Portable & - Portable & - Immediate treatment option \\
\hline & & $\begin{array}{l}\text { - Flow independent } \\
\text { technique }\end{array}$ & - Widely available & - Widely available & $\begin{array}{l}\text { - Gold standard for several } \\
\text { indications and therapeutic } \\
\text { decision making }\end{array}$ \\
\hline & & $\begin{array}{l}\text { - Accuracy } \\
\text { close to DSA }\end{array}$ & & $\begin{array}{l}\text { - Real-time monitoring/ } \\
\text { hemo-dynamic } \\
\text { information }\end{array}$ & \\
\hline & & $\begin{array}{l}\text { - Less motion } \\
\text { artifacts }\end{array}$ & & & \\
\hline \multirow[t]{4}{*}{ Disadvantages } & $\begin{array}{l}\text { - Limited availability } \\
\text { and feasibility } \\
\text { in acute setting }\end{array}$ & - Contrast dependent & $\begin{array}{l}\text { - Operator and } \\
\text { experience dependent }\end{array}$ & $\begin{array}{l}\text { - Operator- and } \\
\text { experience dependent }\end{array}$ & - Contrast dependent \\
\hline & $\begin{array}{l}\text { - Contrast dependent } \\
\text { (CE-MRA) }\end{array}$ & - Radiation exposure & $\begin{array}{l}\text { - Assessment limited } \\
\text { to ICA on the neck } \\
\text { and inter-osseal VA } \\
\text { segments }\end{array}$ & $\begin{array}{l}\text { - Dependent on bone } \\
\text { "window" } \\
\text { without contrast }\end{array}$ & - Radiation exposure \\
\hline & - Flow and motion artifacts & & & & $\begin{array}{l}\text { - Peri- and postprocedural } \\
\text { complications }\end{array}$ \\
\hline & - Inferior accuracy & & & & - Availability still limited \\
\hline
\end{tabular}

CE-MRA = contrast-enhanced magnetic resonance angiography; CTA $=$ computed tomographic angiography; DSA $=$ digital subtraction angiography; ICA = internal carotid artery; MRA = magnetic resonance angiography; TCD = transcranial Doppler; TOF = time-of-flight magnetic resonance angiography; $\mathrm{VA}=$ vertebral artery

rhage, particularly in young patients [22], and to identify the "spot" sign that can select patients at greater risk of hematoma expansion, because contrast extravasation may occur with compromised vessel integrity $[23,24]$.

There are some disadvantages with CTA (Table 2). One of the major concerns is the risk of radio-contrast nephropathy, particularly in patients with renal dysfunction or diabetes. However, a recent published study showed a low incidence of radio-contrast nephropathy in acute stroke patients undergoing emergent CTA, irrespective of baseline creatinine levels [25]. The risk of other immediate adverse reactions after injection of iodinated contrast material seems acceptable [26]. In addition to noncontrast CT, the use of CTA increases radiation dose and multiple examinations can result in a large cumulative dose that may harm patients [27].

\section{Cerebrovascular Ultrasound}

Current ultrasound methods offer fragmented assessment of the cerebrovascular tree. Carotid and vertebral duplex evaluates proximal segments accessible through the soft tissues on the neck, whereas a transcranial ultrasound can detect flow in some portions of the intracranial carotid system, proximal branches of the circle of Willis, and intracranial vertebrobasilar system. In turn, we examine pre-cerebral and intracranial imaging with an ultrasound.

\section{Carotid and Vertebral Duplex}

Extracranial ultrasound can differentiate normal from diseased arteries, identify all categories of stenosis, localize the disease process, including occlusions, detect progressions of the disease, and assess collateral circulation to maintain cerebral blood flow (Table 1). This constitutes a cheap and widely available screening method to diagnose atheromatous disease in the proximal internal carotid artery (ICA) and common carotid artery and to further identify candidates for more expensive or invasive diagnostic evaluations (Table 2 and Fig. 1).

Approximately 1 of 5 ischemic strokes originates from atherothrombotic plaque and stenosis in the carotid arteries [28]. Carotid ultrasound can localize the plaque, and identify its length, composition, and surface. Further Doppler information on peak systolic and end-diastolic velocities, and ICA/common carotid artery ratio is further used to predict the North American carotid artery stenosis range [29]. Although the 2 large carotid endarterectomy trials, the European Carotid Surgery Trial (ECST) and the North American Symptomatic Carotid Endarterectomy Trial (NASCET) applied different methodologies to calculate the percent diameter reduction of the tightest residual lumen $[30,31]$, the current recommendation is that the percent diameter reduction should be measured relative to 
ICA diameter in the disease-free ICA distal to the stenosis (i.e., the North American Symptomatic Carotid Endarterectomy Trial [NASCET] or the North American [N] method). Precise estimation of the stenosis grade is difficult, and ultrasound studies are expected to provide range estimation (i.e., 50-69\%) vs pin-point measurement (i.e., 53.7\%) due to the wide variability of key velocity measurements and the choice of single projection angiographic images to measure the stenosis [29, 32]. Numerous and variable criteria for quantifying the degree of carotid stenosis were introduced in the past 40 years [33-36]. In 2002, the Society of Radiologists in Ultrasound (SRU) convened a multidisciplinary panel that arrived at the SRU Consensus Criteria for grading carotid stenosis with duplex ultrasound (Table 3) [29]. The criteria are recommended for use by new ultrasound laboratories in development and selfvalidating studies of local interpretation criteria, and as a comparator criteria for established laboratories, because in the experience of participating experts, most accurate local criteria should be quite similar (i.e., with a $10-20 \%$ difference from values identified in Table 3). The limitation of the SRU Consensus Criteria is that these are intended for grading a focal and unilateral carotid stenosis only, and these criteria do not offer guidance to grade tandem or bilateral carotid artery disease.

Carotid ultrasound is also a useful tool in the diagnosis of arterial occlusions and some dissections. Although carotid ultrasound is both sensitive and specific to identify proximal atherothrombotic or embolic ICA occlusion [37], the sensitivity of carotid ultrasound for detection of a double lumen and an intimal flap is variable [38]. Intramural hematomas may be missed either when they are located outside the arterial segments directly visible by carotid ultrasound or if they are too small to cause hemodynamically significant stenosis. For this reason, a negative carotid ultrasound requires follow-up with an MRA or DSA if a distal arterial dissection is strongly suspected.

Early re-stenosis after carotid artery stenting or CEA occurs in as much as $10 \%$ of patients [39] and carotid ultrasound remains the primary imaging modality for postintervention surveillance. Analog to the assessment of native carotid arteries, limitations include a high carotid bifurcation, a long ICA plaque, calcification shadows, acoustic artifacts, short neck, presence of tortuosity, and near-occlusion. Furthermore, it should be mentioned that the deployment of a stent alters the compliance and blood flow velocities [40], which make it difficult to use the standard velocity criteria for the evaluation of stented carotid arteries. Thus, in some cases, additional imaging modalities, such as CTA or MRA, may be considered to confirm abnormal findings.

Our ability to diagnose and understand the mechanisms of vertebrobasilar ischemia is less developed compared to the anterior circulation vessels. Vertebral ultrasound allows segmental assessment of the vertebral arteries often obscured by transverse processes and deep location of the vertebral origins. If successful, ultrasound can detect stenoses of the vertebral arteries and less commonly detect the presence of atherosclerotic plaques, intraluminal thrombi, or a double lumen with an extracranial vertebral dissection $[38,41]$. Fewer validation studies are available to derive diagnostic criteria, and generally the accuracy parameters for vertebral ultrasound are less than these of carotid duplex.

\section{Transcranial Doppler and Transcranial Duplex}

Although the term imaging refers mostly to transcranial duplex technology and its ability to generate color or power flow images, both transcranial Doppler (TCD) and duplex terms refer to the assessment of the proximal cerebral vasculature through spectral Doppler sampling for flow velocity and waveforms. Specific descriptions of ultrasound technology and scanning protocols can be found elsewhere $[42,43]$.

TCD became the standard of care at comprehensive stroke centers as being one of the essential diagnostic tests and services that a modern stroke team should have at their disposal [44]. Current clinical indications and expected outcomes were recently summarized by the multidisciplinary panel of the American Society of Neuroimaging Practice Guidelines Committee (Table 1) [45].

Table 3 The Society of Radiologists in Ultrasound consensus criteria for carotid stenosis

\begin{tabular}{lllll}
\hline Stenosis range N method & ICA PSV & ICA/CCA PSV ratio & ICA EDV & Plaque \\
\hline Normal & $<125 \mathrm{~cm} / \mathrm{s}$ & $<2.0$ & $<40 \mathrm{~cm} / \mathrm{s}$ & None \\
$<50 \%$ & $<125 \mathrm{~cm} / \mathrm{s}$ & $<2.0$ & $<40 \mathrm{~cm} / \mathrm{s}$ & $<50 \%$ diameter reduction \\
$50-69 \%$ & $125-230 \mathrm{~cm} / \mathrm{s}$ & $2.0-4.0$ & $40-100 \mathrm{~cm} / \mathrm{s}$ & $>50 \%$ diameter reduction \\
$70-$ near occlusion & $>230 \mathrm{~cm} / \mathrm{s}$ & $>4.0$ & $>100 \mathrm{~cm} / \mathrm{s}$ & $>50 \%$ diameter reduction \\
Near occlusion & May be low or undetectable & Variable & Variable & Significant, detectable lumen \\
Occlusion & Undetectable & Not applicable & Not applicable & Significant, no detectable lumen \\
\hline
\end{tabular}

$\mathrm{EDV}=$ end diastolic velocity; ICA/CCA = internal carotid artery/common carotid artery; PSV = peak systolic velocity 
TCD offers complementary information to MRA and CTA in the assessment of cerebral ischemia [46, 47]. Patients with symptomatic or asymptomatic high-grade ICA stenosis can undergo TCD monitoring to detect, localize, and quantify cerebral embolization [48]. This information is helpful to establish the diagnosis and change management strategy, if emboli are found pointing to a potential proximal source of embolism or continuing embolization that identifies the patients at highest risk of recurrent or first-ever stroke [49]. The presence of emboli on TCD distal to a high-grade asymptomatic ICA stenosis identifies patients at higher risk of first-ever stroke and may be a useful risk predictor for identifying those asymptomatic carotid stenosis patients who might benefit from intervention with CEA [49]. In patients with symptomatic carotid stenosis, combination therapy with aspirin and clopidogrel is more effective than monotherapy in reducing asymptomatic embolization [50, 51]. Moreover, detection of emboli on TCD can be the only sign of a proximal arterial dissection, partially occlusive thrombus or an unrecognized cardiac source of embolism, and may identify paradoxic embolization through a cardiac right-toleft shunt (during intravenous injection of agitated normal saline) [46, 52].

Intracranial MRA often shows flow gaps due to turbulence or reversal of flow direction, thus overestimating the degree of an arterial stenosis. TCD findings of the residual flow signals, abnormal waveforms, reversed flow direction, collateral channels, and flow diversion to a branching vessel confirm the presence of an intracranial steno-occlusive lesion in real time, and validated diagnostic criteria are available (Fig. 1) [36, 41]. The Thrombolysis in Brain Ischemia (TIBI) flow grading system was developed to evaluate residual flow on TCD and to monitor thrombus dissolution in real time [53], which can be helpful in prediction of stroke severity and mortality, as well as likelihood of re-canalization and clinical improvement [54]. Of note, the yield of TCD is greatest the closer in time it is performed to stroke symptom onset, and it is higher in anterior than posterior circulation [55]. Notably, power motion mode Doppler TCD or imaging with transcranial color-coded duplex increase the diagnostic accuracy of ultrasound tests for the assessment of vertebrobasilar circulation [56].

TCD can reliably rule out a proximal intracranial stenosis according to the findings of the recently published Stroke Outcomes and Neuroimaging of Intracranial Atherosclerosis (SONIA) trial, which aimed to define the positive and negative predictive value of TCD/MRA for the identification of 50 to $99 \%$ intracranial stenosis in the intracranial ICA, middle cerebral artery, vertebral artery, and basilar artery [57]. However, abnormal findings on TCD or MRA require a confirmatory test, such as catheter angiography, to reliably identify the degree of intracranial stenoses.
In addition to vessel surveillance, TCD testing can include assessment of vasomotor reactivity, which may be of interest for providing a more comprehensive indication to surgical treatment in subgroups of subjects with symptomatic and asymptomatic carotid stenosis and occlusions [58, 59]. This information is not available from carotid duplex ultrasound examination and may require additional contrast studies with magnetic resonance perfusion or Diamox single photon emission computed tomography or perfusion CT that are costly, and may lead to complications related to vasoactive Diamox effects that last longer than a brief hypercapnia with breath holding.

Numerous studies showed the effectiveness of TCD in diagnosing cerebral vasospasm, both in the anterior and posterior circulation after SAH, allowing early planning of interventions, including angioplasty and nicardipine infusions [60, 61]. A detailed review of this application of TCD and the role of CTA in SAH is largely outside the scope of our article, which focuses mostly on ischemic stroke.

Continuous TCD monitoring during intra-arterial rescue procedures may detect potentially harmful flow changes like re-occlusion, hypo- and hyper-perfusion or thromboembolism and air embolization, and it can be helpful particularly when neurological assessment in patients is limited because of sedation and intubations [62].

In summary, TCD provides a noninvasive and inexpensive (relative to angiography) monitoring modality that can be used in a variety of clinical situations to provide real-time physiological information that is often unobtainable with other testing, without increasing patient risks (i.e., repeated radiation doses, contrast injections) and associated costs (Table 2). The main disadvantage of TCD and duplex imaging is that these tests are extremely operator-dependent and the skilled sonographers and clinician users of these technologies are few in number to meet the need of stroke patients.

\section{Invasive Vascular Imaging}

\section{DSA}

Two large, randomized, clinical trials, the NASCET and the European Carotid Surgery Trial (ECST), demonstrated the benefit of CEA in patients with high-grade (70-99\%) symptomatic carotid artery stenosis [30, 31]. Thus, the accurate diagnosis of high-grade stenosis remains critical for selection of stroke patients who are amenable to CEA. The severity of stenosis in the endarterectomy trials was evaluated by DSA, which has become the gold standard for selecting patients for CEA, and recently for deciding between CEA and stenting (Table 1).

DSA, however, has a risk of peri- and post-procedural complications (e.g., embolism, dissections, retroperitoneal 
hematoma, allergic reactions to contrast medium, nephropathy), particularly in older patients with polyvascular atherosclerosis, resulting in a morbidity and mortality from 1 to $4 \%[62,63]$. In the ACAS trial of patients with asymptomatic carotid artery stenosis, half of early stroke risk was attributed to performance of diagnostic catheter angiography [64]. Even in patients without new neurological symptoms after DSA, minor asymptomatic infarctions could be apparent on cerebral imaging, presumably as a consequence of embolism [65].

The question as to whether or not to perform CEA could be solely based on carotid duplex ultrasound or on the combination of noninvasive vascular imaging modalities. In a comparative study by Patel et al. [66], carotid ultrasound, CTA, MRA, and DSA were performed in patients with symptomatic high-grade carotid stenosis. The differentiation from operable and nonoperable carotid artery lesions was achieved with ultrasound (most closely), CTA (tendency to underestimation), and MRA (tendency to overestimation) in a majority of studied patients comparable with DSA. However, a comparable diagnostic accuracy was only achieved when 2 noninvasive tests showed congruent results; in disagreement, the third modality had to also be taken into account to make an accurate judgment as compared with DSA. Therefore, no technique on its own appears accurate enough to replace DSA to determine the severity of a carotid artery stenosis amenable for CEA (Fig. 1). The current guidelines for acute ischemic stroke recommend carotid ultrasound as a screening technique for imaging the pre-cerebral vasculature, whereas a confirmatory study with a second modality, such as CE-MRA or CTA seems justified, particularly when making decisions regarding invasive therapies [67].

The interest in performance of diagnostic DSA and development on newer catheters aiding neurovascular therapies is now fueled by proliferation of intra-arterial rescue procedures offered to stroke patients by the growing number of neuroendovascular trained specialists. DSA is increasingly used in the diagnostic work-up and management of acute ischemic stroke patients, emerging as an important therapeutic tool [68]. To further support the use of DSA in routine practice, a randomized efficacy phase III trial is needed to confirm the promise of intra-arterial rescue shown in the Prolyse in Acute Cerebral Thromboembolism (PROACT) II study [69].

DSA has been the preferred follow-up imaging modality for detecting in-stent re-stenosis after intracranial stenting, which constitutes a frequent long-term sequela [70]. Although less invasive techniques for follow-up investigations would be desirable (particularly in patients at high risk for in-stent re-stenosis), noninvasive imaging with TCD, CTA, and MRA is potentially affected by stent-related artifacts and hemodynamic changes.
Limitations of DSA include its operator dependency and availability because cerebral angiography requires specific and intensive training (Table 2) [71]. However, the future progress of stroke care lies in the establishment of hub-andspokes networks to reach the population of patients with established reperfusion therapy, such as IPA and to aid the transfer of patients in need of comprehensive stroke care [44] to the centers with subspecialty trained clinicians. Catheter angiography and expert use of advanced vascular imaging technologies will play a pivotal role in this process.

Acknowledgments Full conflict of interest disclosure is available in the electronic supplementary material for this article.

\section{References}

1. Turski PA, Korosec F. MR angiography of the head and neck; in Edelman RR, Hesselink JR, Zlatkin MB, Crues JV III, eds. Clinical Magnetic Resonance Imaging. Philadelphia: SaundersElsevier, 2006;2:1499-1537.

2. Bash S, Villablanca JP, Jahan R. Intracranial vascular stenosis and occlusive disease: evaluation with $\mathrm{CT}$ angiography, MR angiography, and digital subtraction angiography. AJNR Am J Neuroradiol 2005;26:1012-1021.

3. Korosec F, Turski P. Velocity and volume flow measurements using phase-contrast magnetic resonance imaging. Int J Neuroradiol 1997;3:293-318.

4. Debrey SM, Yu H, Lynch JK, et al. Diagnostic accuracy of magnetic resonance angiography for internal carotid artery disease: a systematic review and meta-analysis. Stroke 2008;39:2237-2248.

5. Phan T, Huston J III, Bernstein MA, Riederer SJ, Brown RD Jr. Contrast-enhanced magnetic resonance angiography of the cervical vessels: experience with 422 patients. Stroke 2001;32:2282-2286.

6. Provenzale JM, Sarikaya B. Comparison of test performance characteristics of MRI, MR angiography, and CT angiography in the diagnosis of carotid and vertebral artery dissection: a review of the medical literature. AJR Am J Roentgenol 2009;193:1167-1174.

7. Berletti R, Cavagna E, Cimini N, Moretto G, Schiavon F. Dissection of epiaortic vessels: clinical appearance and potentiality of imaging techniques. Radiol Med 2004;107:35-46.

8. Hand PJ, Wardlaw JM, Rowat AM. Magnetic resonance brain imaging in patients with acute stroke: feasibility and patient related difficulties. J Neurol Neurosurg Psychiatry 2005;76:1525-1527.

9. Weinreb JC, Abu-Alfa AK. Gadolinium-based contrast agents and nephrogenic systemic fibrosis: why did it happen and what have we learned? J Magn Reson Imaging 2009;30:1236-1239.

10. Schellinger PD: The evolving role of advanced MR imaging as a management tool for adult ischemic stroke: a Western-European perspective. Neuroimaging Clin North Am 2005;15:245-258.d

11. Tomandl BF, Klotz E, Handschu R, et al. Comprehensive imaging of ischemic stroke with multisection CT. Radiographics 2003;23:565-592

12. Vieco PT. CT angiography of the intracranial circulation. Neuroimaging Clin N Am 1998;8:577-592.

13. Blank M, Kalender WA. Medical volume exploration: gaining insights virtually. Eur J Radiol 2000;33:161-169.

14. Lell MM, Anders K, Uder M, et al. New techniques in CT angiography. Radiographics 2006;26:45-62. 
15. Bash S, Villablanca JP, Jahan R, et al. Intracranial vascular stenosis and occlusive disease: evaluation with CT angiography, MR angiography, and digital subtraction angiography. AJNR Am J Neuroradiol 2005;26:1012-21.

16. Puetz V, Dzialowski I, Hill MD. Calgary CTA Study Group. Intracranial thrombus extent predicts clinical outcome, final infarct size and hemorrhagic transformation in ischemic stroke: the clot burden score. Int J Stroke 2008;3:230-236.

17. Saqqur M, Uchino K, Demchuk AM. CLOTBUST Investigators. Site of arterial occlusion identified by transcranial Doppler predicts the response to intravenous thrombolysis for stroke. Stroke 2007;38:948-954.

18. Schramm P, Schellinger PD, Fiebach JB, et al. Comparison of CT and CT angiography source images with diffusion-weighted imaging in patients with acute stroke within 6 hours after onset. Stroke 2002;33:2426-2432.

19. Wright AR, Gibson RJ, Sellar RJ. Outcome, observer reliability, and patient preferences if CTA, MRA, or Doppler ultrasound were used, individually or together, instead of digital subtraction angiography before carotid endarterectomy. J Neurol Neurosurg Psychiatry 2002;73:21-28.

20. Lev MH, Romero JM, Goodman DN, et al. Total occlusion versus hairline residual lumen of the internal carotid arteries: accuracy of single section helical CT angiography. AJNR Am J Neuroradiol 2003;24:1123-1129.

21. Papke K, Kuhl CK, Fruth M, et al. Intracranial aneurysms: role of multidetector CT angiography in diagnosis and endovascular therapy planning. Radiology 2007;244:532-540.

22. Morgenstern LB, Hemphill JC 3 rd, Anderson C, et al. American Heart Association Stroke Council and Council on Cardiovascular Nursing. Guidelines for the management of spontaneous intracerebral hemorrhage: a guideline for healthcare professionals from the American Heart Association/American Stroke Association. Stroke 2010;41:2108-2129.

23. Delgado Almandoz JE, Yoo AJ, et al. Systematic characterization of the computed tomography angiography spot sign in primary intracerebral hemorrhage identifies patients at highest risk for hematoma expansion: the spot sign score. Stroke 2009;40:2994 3000 .

24. Wada R, Aviv RI, Fox AJ, et al. CT angiography "spot sign" predicts hematoma expansion in acute intracerebral hemorrhage. Stroke 2007;38:1257-1262.

25. Krol AL, Dzialowski I, Roy J, et al. Incidence of radiocontrast nephropathy in patients undergoing acute stroke computed tomography angiography. Stroke 2007;38:2364-2366.

26. Shrier DA, Tanaka H, Numaguchi Y, Konno S, Patel U, Shibata D. CT angiography in the evaluation of acute stroke. AJNR Am J Neuroradiol 1997;18:1011-1020.

27. Berrington de González A, Mahesh M, Kim KP, et al. Projected cancer risks from computed tomographic scans performed in the United States in 2007. Arch Intern Med 2009;169:2071-2077.

28. Hennerici M, Hulsbomer HB, Hefter H, et al. Natural history of asymptomatic extracranial arterial disease: results of a long-term prospective study. Brain 1987;110:777-791.

29. Grant EG, Benson CB, Moneta GL, et al. Carotid artery stenosis: grayscale and Doppler ultrasound diagnosis - Society of Radiologists in Ultrasound consensus conference. Ultrasound Q 2003;19:190-198.

30. European Carotid Surgery Trialists' Collaborative Group. MRC European Carotid Surgery Trial: interim results for symptomatic patients with severe $(70-99 \%)$ or with mild $(0-29 \%)$ carotid stenosis. Lancet 1991;337:1235-1243.

31. North American Symptomatic Carotid Endarterectomy Trial Collaborators. Beneficial effect of carotid endarterectomy in symptomatic patients with high grade carotid stenosis. New Engl J Med 1991;325:445-453.
32. Rothwell PM, Gibson RJ, Slattery J, Sellar RJ, Warlow CP. Equivalence of measurements of carotid stenosis. A comparison of three methods on 1001 angiograms. Stroke 1994;25:24352439.

33. Aburahma AF, Srivastava M, Stone PA, et al. Critical appraisal of the Carotid Duplex Consensus criteria in the diagnosis of carotid artery stenosis. J Vasc Surg 2010;14:xx.

34. Beach KW, Bergelin RO, Leotta DF, et al. Standardized ultrasound evaluation of carotid stenosis for clinical trials: University of Washington Ultrasound Reading Center. Cardiovasc Ultrasound 2010;8:39.

35. Widder B, von Reutern GM, Neuerburg-Heusler D. Morphologic and Doppler sonographic criteria for determining the degree of stenosis of the internal carotid artery. Ultraschall Med 1986;7:70-75.

36. Spencer MP, Reid JM. Quantitation of carotid stenosis with continuous-wave (C-W) Doppler ultrasound. Stroke 1979;10:32630.

37. Garami Z, Calleja S, Labiche L, Chernyshev OY, Campbell M, Malkoff M, Alexandrov AV Yield of urgent carotid scanning in acute cerebral ischemia. Stroke 2003;34:266.

38. Schievink WI. Spontaneous dissection of the carotid and vertebral arteries. N Engl J Med 2001;344:898-906.

39. Gröschel K, Riecker A, Schulz JB, Ernemann U, Kastrup A. Systematic review of early recurrent stenosis after carotid angioplasty and stenting. Stroke 2005;36:367-373.

40. Mathur A, Dorros G, Iyer SS, Vitek JJ, Yadav SS, Roubin GS. Palmaz stent compression in patients following carotid artery stenting. Cathet Cardiovasc Diagn 1997;41:137-140.

41. Khan S, Cloud GC, Kerry S, Markus HS. Imaging of vertebral artery stenosis: a systematic review. J Neurol Neurosurg Psychiatry $2007 ; 78: 1218-1225$.

42. Alexandrov AV, Sloan MA, Wong LK, et al, American Society of Neuroimaging Practice Guidelines Committee. Practice Guidelines Committee. Practice standards for transcranial Doppler ultrasound: part I-test performance. J Neuroimaging 2007;17:11-8.

43. Nedelmann M, Stolz E, Gerriets T, et al, TCCS Consensus Group. Consensus recommendations for transcranial color-coded duplex sonography for the assessment of intracranial arteries in clinical trials on acute stroke. Stroke 2009;40:3238-3244.

44. Alberts MJ, Latchaw RE, Selman WR, et al, for the Brain Attack Coalition. Recommendations for comprehensive stroke centers: a consensus statement from the brain attack coalition. Stroke 2005;36:1597-1618.

45. Alexandrov AV, Sloan MA, Tegeler $\mathrm{CH}$, et al, for the American Society of Neuroimaging Practice Guidelines Committee. Practice Standards for Transcranial Doppler (TCD) Ultrasound. Part II. Clinical Indications and Expected Outcomes. J Neuroimaging 2010;26:xx

46. Ritter MA, Dittrich R, Thoenissen N, et al. Prevalence and prognostic impact of microembolic signals in arterial sources of embolism. A systematic review of the literature. J Neurol 2008;255:953-961.

47. Markus HS, King A, Shipley M, et al. Asymptomatic embolisation for prediction of stroke in the Asymptomatic Carotid Emboli Study (ACES): a prospective bservational study. Lancet Neurol 2010;9:663-671.

48. Wijman CA, McBee NA, Keyl PM, et al. Diagnostic impact of early transcranial Doppler ultrasonography on the TOAST classification subtype in acute cerebral ischemia. Cerebrovasc Dis 2001;11:317-323.

49. Tsivgoulis G, Sharma VK, Lao AY, Malkoff MD, Alexandrov AV. Validation of transcranial Doppler with computed tomography angiography in acute cerebral ischemia. Stroke 2007;38:1245-1249.

50. Markus HS, Droste DW, Kaps M, et al. Dual antiplatelet therapy with clopidogrel and aspirin in symptomatic carotid stenosis evaluated using doppler embolic signal detection: the Clopidogrel 
and Aspirin for Reduction of Emboli in Symptomatic Carotid Stenosis (CARESS) trial. Circulation 2005;111:2233- 2240.

51. Wong KS, Chen $\mathrm{C}, \mathrm{Fu}$ J, et al, for the CLAIR study investigators. Clopidogrel plus aspirin versus aspirin alone for reducing embolisation in patients with acute symptomatic cerebral or carotid artery tenosis (CLAIR study): a randomised, open label, blinded endpoint trial. Lancet Neurol 2010;9:489-497.

52. Lao AY, Sharma VK, Tsivgoulis G, et al. Detection of right-to-left shunts: comparison between the International Consensus and Spencer Logarithmic Scale criteria. J Neuroimaging 2008;18:402-406.

53. Demchuk AM, Burgin WS, Christou I, et al. Thrombolysis in brain infarction (TIBI) transcranial Doppler flow grades predict clinical severity, early recovery, and mortality in patients treated with intravenous tissue plasminogen activator. Stroke 2001;32:89-93.

54. Tsivgoulis G, Sharma VK, Lao AY, et al. Validation of transcranial Doppler with computed tomography angiography in acute cerebral ischemia. Stroke 2007;38:1245-1249.

55. Alexandrov AV, Demchuk A, Wein T, et al. The yield of transcranial Doppler in acute cerebral ischemia. Stroke 1999;30:1605-1609.

56. Tsivgoulis G, Sharma VK, Hoover SL, et al. Applications and advantages of power motion-mode Doppler in acute posterior circulation cerebral ischemia. Stroke 2008;39:1197-1204.

57. Feldmann E, Wilterdink JL, Kosinski A, et al, Stroke Outcomes and Neuroimaging of Intracranial Atherosclerosis (SONIA) Trial Investigators. The Stroke Outcomes and Neuroimaging of Intracranial Atherosclerosis (SONIA) trial. Neurology 2007;68:2099-2106.

58. Silverstrini M, Vernieri F, Pasqualetti $P$, et al. Impaired vasosmotor reactivity and risk of stroke in patients with asymptomatic carotid artery stenosis. JAMA 2000;283:2122-2127.

59. Vernieri F, Pasqualetti P, Matheis M, et al. Effect of collateral flow and cerebral vasomotor reactivity on the outcome of carotid artery occlusion. Stroke 2001;32:1552-1558.

60. Sloan MA, Haley EC Jr, Kassell NF, et al. Sensitivity and specificity of transcranial Doppler ltrasonography in the diagnosis of vasospasm following subarachnoid hemorrhage. Neurology 1989;39:1514-1518.

61. Sloan MA, Burch CM, Wozniak MA, et al. Transcranial Doppler detection of vertebrobasilar vasospasm following subarachnoid hemorrhage. Stroke 1994;25:2187-2197.

62. Rubiera M, Cava L, Tsivgoulis G, et al. Diagnostic criteria and yield of real-time transcranial Doppler monitoring of intra-arterial reperfusion procedures. Stroke 2010;41:695-699.
63. Hankey GJ, Warlow CP, Molyneux AJ. Complications of cerebral angiography for patients with mild carotid territory ischaemia being considered for carotid endarterectomy. J Neurol Neurosurg Psychiatry 1990;53:542-548.

64. Endarterectomy for asymptomatic carotid artery stenosis. Executive Committee for the Asymptomatic Carotid Atherosclerosis Study. JAMA 1995;273:1421-1428.

65. Bendszus M, Koltzenburg M, Burger R, Warmuth-Metz M, Hofmann E, Solymosi L. Silent embolism in diagnostic cerebral angiography and neurointerventional procedures: a prospective study. Lancet 1999;354:1594-1597.

66. Patel SG, Collie DA, Wardlaw JM. Outcome, observer reliability, and patient preferences if CTA, MRA, or Doppler ultrasound were used, individually or together, instead of digital subtraction angiography before carotid endarterectomy. J Neurol Neurosurg Psychiatry 2002;73:21-28.

67. Latchaw RE, Alberts MJ, Lev MH, et al. American Heart Association Council on Cardiovascular Radiology and Intervention, Stroke Council, and the Interdisciplinary Council on Peripheral Vascular Disease. Recommendations for imaging of acute ischemic stroke: a scientific statement from the American Heart Association. Stroke 2009;40:3646-3678.

68. Broderick JP. Endovascular therapy for acute ischemic stroke. Stroke 2009;40:103-106.

69. Furlan A, Higashida R, Wechsler L, et al. Intra-arterial prourokinase for acute ischemic stroke. The PROACT II study: a randomized controlled trial. Prolyse in Acute Cerebral Thromboembolism. JAMA 1999;282:2003-2011.

70. Zaidat OO, Klucznik R, Alexander MJ, et al. NIH Multi-center Wingspan Intracranial Stent Registry Study Group. The NIH registry on use of the Wingspan stent for symptomatic 70-99\% intracranial arterial stenosis. Neurology 2008;70:1518 -1524.

71. Connors JJ 3rd, Sacks D, Furlan AJ, et al. Training, competency, and credentialing standards for diagnostic cervicocerebral angiography, carotid stenting, and cerebrovascular intervention: a joint statement from the American Academy of Neurology, the American Association of Neurological Surgeons, the American Society of Interventional and Therapeutic Neuroradiology, the American Society of Neuroradiology, the Congress of Neurological Surgeons, the AANS/CNS Cerebrovascular Section, and the Society of Interventional Radiology. J Vasc Interv Radiol 2009;20: S292-S301 